\title{
Structure and electronic structure of functional materials under symmetric breaking
}

\author{
$\operatorname{Lin} \mathrm{Gu}^{1 *}$ \\ 1. The Institute of Physics, Chinese Academy of Sciences, Beijing, China \\ * Corresponding author: 1.gu@ iphy.ac.cn
}

Introduction

The origin of functional materials is divided into two parts, multi-electronic system and single electronic system. Multi-electronic system is determined by various fields, while single electronic system is determined by local symmetry. From Noether's vision, to every differentiable symmetry generated by local actions there corresponds a conserved flux. Materials with broken symmetry destroy the conserved quantities and therefore have functionality. Even the change under picometer scale in functional materials could break the symmetry. As a powerful characterization method, Spherical aberration corrected electron microscopy could acquire information about atomic-scale structure and electronic structure, which could get rid of the structure-activity relationship under the constraint condition of periodic potential field, single electron approximation, and adiabatic approximation theories in solid material. In our recent work, we realized broken symmetry in functional material by electrochemical operation [1][2]. For example, the interface regions, including surfaces and internal boundaries, may have a substantial impact on battery performance. Both the spatial inversion symmetry and translational symmetry are broken by the interfacial structure itself (Figure 2). This report will focus on electrochemical operation of functional oxide materials, physical mechanism of electrochemical energy storage materials, and electron structure of catalyst materials, to discuss the correlation between microstructure and electronic structure in the broken symmetric system.

Material \& Methods

For the first time, we use a state-of-the-art chip based in situ TEM holder, in combination with focused ion beam (FIB) milling, to prepare a working all-solid-state LIB sample (Figure 1). A set of optimized FIB milling procedures and beam conditions was used to minimize ion-beam damage and assemble the battery efficiently. These procedures and conditions are detailed in our publication [1][2].

\section{Results \& Discussion}

In summary, we designed a working all-solid-state lithium-ion battery in TEM and directly observed the formation of nanopolycrystallization on an atomic scale by using aberration-corrected STEM. A battery consisting of $\mathrm{LiCoO}_{2}$ cathode, LLZO solid state electrolyte and gold anode was constructed, delithiated and observed in an aberration corrected scanning transmission electron microscope at atomic scale (Figure 1A). We found that the pristine single crystal $\mathrm{LiCoO}_{2}$ became nanosized polycrystal connected by coherent twin boundaries and antiphase domain boundaries after high voltage delithiation. This is different from liquid electrolyte batteries, where a series of phase transitions take place at $\mathrm{LiCoO}_{2}$ cathode during delithiation. Both grain boundaries become more energy favorable along with extraction of lithium ions through theoretical calculation [1]. Besides, in our follow-on work, we used the similar methods to develop more details on the sample fabricated by FIB milling (Figure 1B). The in-situ studies $\mathrm{LiNi}_{0.5} \mathrm{Mn}_{1.5} \mathrm{O}_{4}$ from various zone axes reveal the evolution of both atomic and electronic structures during delithiation, which is found due to the migration of oxygen and transition-metal ions. Ordered to disordered structural transition proceeds along the $\langle 100\rangle,\langle 110\rangle,\langle 111\rangle$ directions and 
inhomogeneous structural evolution along the $\langle 112\rangle$ direction. Uneven extraction of lithium ions leads to localized migration of transition-metal ions and formation of antiphase boundaries. Dislocations facilitate transition-metal ions migration as well. Theoretical calculations suggest that doping of lower valence-state cations effectively stabilize the structure during delithiation and inhibit the formation of boundaries. This study shows the importance of atomic scale three-dimensional characterizations for improving our understanding on the dynamic process and fundamental mechanisms of delithiation [2].

\section{References:}

[1] Yue Gong et al., Journal of the American Chemical Society 139 (2017), p. 4274-4277.

[2] Yue Gong et al., Nature Communications 9 (2018), p. 1-8.

[3] These works were supported by National Program on Key Basic Research Project (2014CB921002), National Natural Science Foundation of China (51522212, 51421002, 51672307), The Key Research Program of Frontier Sciences, CAS (Grant No. QYZDB-SSWJSC035), The Strategic Priority Research Program of Chinese Academy of Sciences (Grant No. XDB07030200), and Beijing Natural Science Foundation (B) (KZ201610005003)
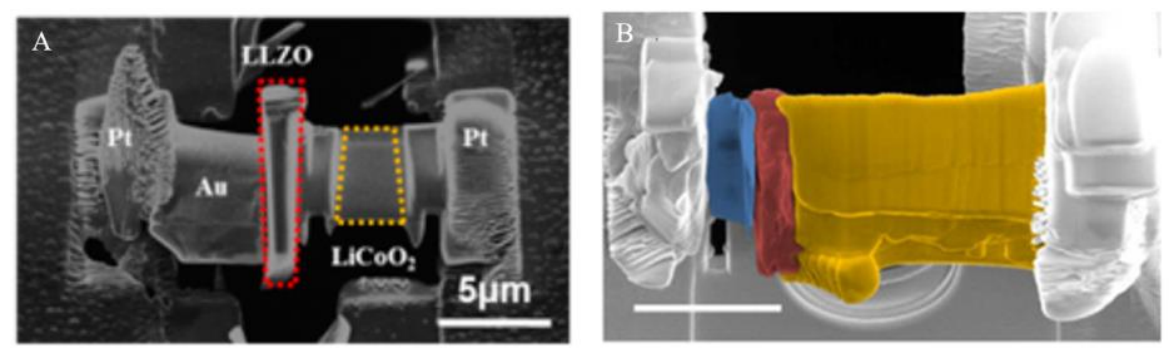

Figure 1. (A) SEM image of the FIB fabricated battery on the nanochip to apply the electric field, and corresponding schematic [1]. (B) SEM image of the assembled all-solid-state lithium-ion battery [2].
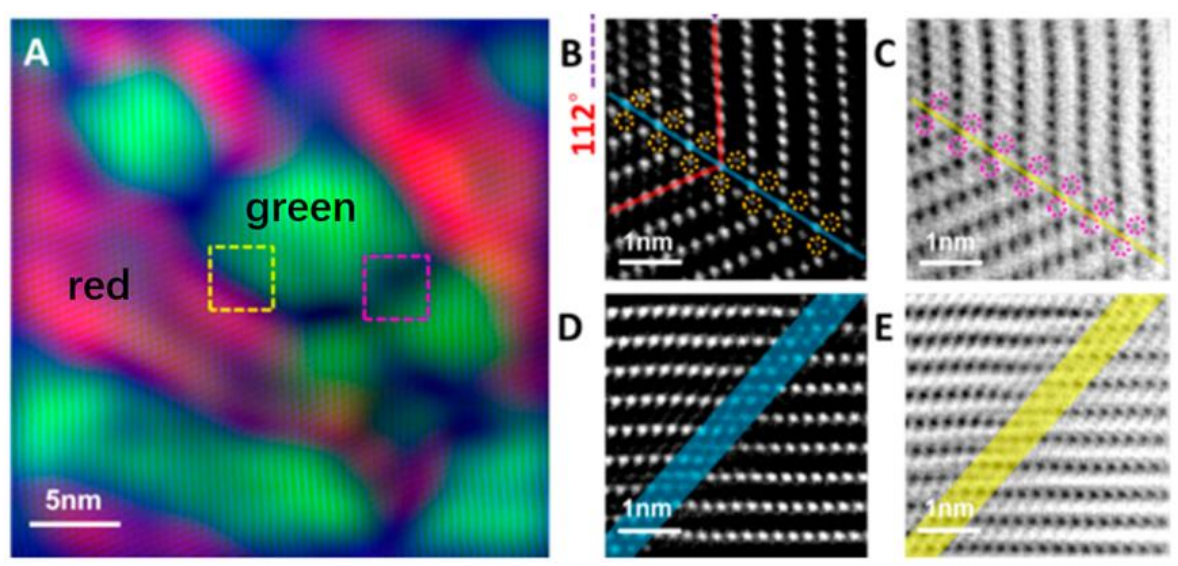

Figure 2. (A) HAADF micrograph of the delithiated $\mathrm{LiCoO}_{2}$ cathode colored using the GPA method. HAADF micrograph colored in blue, and two orientations colored with green and red. Panels B and C are zoomed-in micrographs of the yellow, dashed-line, rectangular area (on the left side of panel A). Panels D and E are zoomed-in micrographs of the pink, dashed-line, rectangular area (on the right side of panel A). For both boundaries, we can see a contrast in the lithium layer in both the HAADF and the $\mathrm{ABF}$ micrograph, which suggests heavy atoms are present in the lithium layer. In this case, it is cobalt ions because of the phase transition in the layer structure, spinel, and rock salt. Furthermore, in panels B and $\mathrm{C}$ we can see that the basal planes of the two crystals meet at an angle of $112^{\circ}$. The angle is $109.5^{\circ}$ in pristine $\mathrm{LiCoO}_{2} \cdot[1]$ 